\title{
Multi-slit emittance measurement study for BNL ERL
}

\section{Liu, D. Gassner, M. Minty, P. Thieberger}

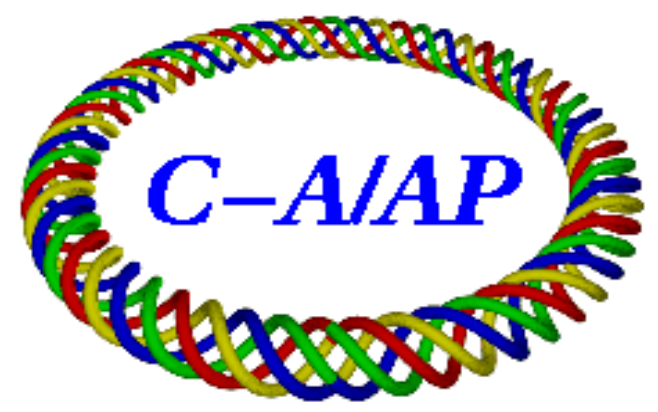

\section{Collider-Accelerator Department Brookhaven National Laboratory Upton, NY 11973}

Notice: This document has been authorized by employees of Brookhaven Science Associates, LLC under Contract No. DE-AC02-98CH10886 with the U.S. Department of Energy. The United States Government retains a nonexclusive, paid-up, irrevocable, world-wide license to publish or reproduce the published form of this document, or allow others to do so, for United States Government purposes. 


\title{
Multi-slit Based Emittance Measurement Study for BNL ERL
}

\author{
C. Liu* and D. Gassner, M. Minty, P. Thieberger \\ BNL, Upton, NY, U.S.A.
}

(Dated: October 12, 2012)

\begin{abstract}
A code for determining the beam emittance from a multi-slit image has been developed. To verify its validity, we simulated a beam distribution in $4 \mathrm{D}$ phase space at the multi-slit position and the resulting image at a downstream profile measurement device. We applied the algorithm to this image pattern to recover the beam emittance at the slit position. The dependence of the relative difference of the inferred emittance and the input emittance on the slit width and drift length are studied in detail and presented in this report.
\end{abstract}

\section{INTRODUCTION}

At the BNL ERL, a multi-slit device will be used for direct measurement of two dimensional beam phase space, which samples particles at discrete horizontal (or vertical) positions and projects the sampled beam on a downstream screen. The beam size and divergence of the sampled beam can be determined using the image acquired by the downstream profile device and the mask pattern dimensions. The emittance of the beamlets sampled by the multi-slit mask will be shown to be representative of the emittance of the beam incident on the mask. Here we will present the algorithm and its application using simulated data, and a study of geomatry-dependent systematic errors in the inferred emittance.

\section{ALGORITHM}

The schematic for a multi-slit emittance measurement device is shown in Fig. 1. The incoming beam is sampled by a multi-slit mask. Beamlets formed by the mask project onto a profile measurement device and the resulting image is recorded. The beam size at the slits can be determined by the dimension of the slit pattern and the intensity of the beamlets. The beam divergence can be determined by the intensity distribution on the profiler.

The well accepted rules for multi-slit mask design $[1,2]$ are: the slit spacing is chosen such that beam is divided to $4-8$ beamlets; the slit width is as narrow as possible as limited by current fabrication techniques; the drift length between mask and profiler is such that beamlets will spread out on the profiler until they overlapping; the mask thickness is thick enough to stop most incoming particles; and the opening angle of the slit is comparable to the anticipated beam divergence.

Detailed formulas for slits emittance measurement can be found in Ref. [3]. The projection of the recorded image is usually treated as a multi-Gaussian distribution. The average divergence of each beamlet is calculated based on slit positions and peak positions. The rms diver-

\footnotetext{
*cliu1@bnl.gov
}

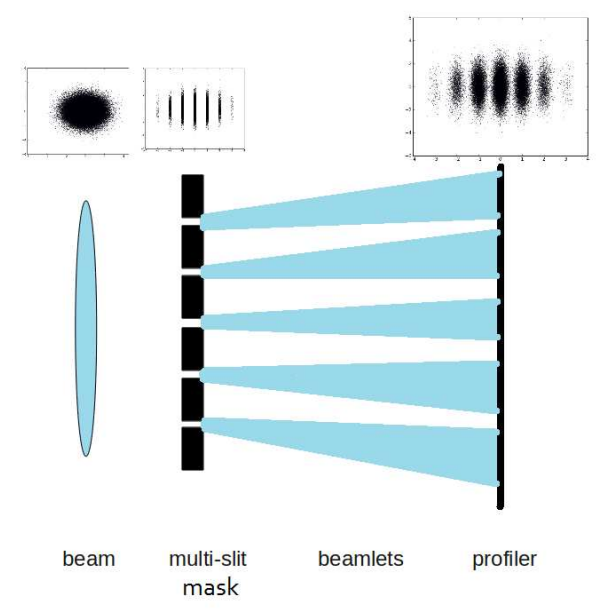

FIG. 1. Schematic of a multi-slit emittance measurement device (not to scale)

gence of the beamlets is calculated from the rms of the fitted Gaussian peaks. Suppose we get three arrays of parameters from multi-Gaussian fits of the projection, $\mathrm{a}, \mathrm{b}$ and $\mathrm{c}$, which are the intensity, peak position and sigma of the peaks. The slit positions are represented by an array $x$. The centroid of the beam at the mask is $<x>=\frac{1}{\sum_{i=1}^{n} a_{i}} \sum_{i=1}^{n}\left(a_{i} * x_{i}\right)$, here $n$ is the number of beamlets. The rms size at the mask is

$$
<x^{2}>=\frac{1}{\sum_{i=1}^{n} a_{i}} \sum_{i=1}^{n}\left(a_{i} *\left(x_{i}-<x>\right)^{2}\right)
$$

The average divergence of the beamlets is given by $x_{i}^{\prime}=$ $\left(b_{i}-x_{i}\right) / L$. The average divergence of the sampled beam is $\left\langle x^{\prime}\right\rangle=\frac{1}{\sum_{i=1}^{n} a_{i}} \sum_{i=1}^{n}\left(a_{i} * x_{i}^{\prime}\right)$. The rms divergence of the beamlets is $\sigma_{i}^{\prime}=c_{i} / L$. The rms divergence of the sampled beam at the mask is

$$
<x^{\prime 2}>=\frac{1}{\sum_{i=1}^{n} a_{i}} \sum_{i=1}^{n}\left(a_{i} *{\sigma_{i}^{\prime}}^{2}+a_{i} *\left(\sigma_{i}^{\prime}-<x^{\prime}>\right)^{2}\right)
$$

The position angle correlation term is

$$
<x x^{\prime}>=\frac{1}{\sum_{i=1}^{n} a_{i}}\left(\sum_{i=1}^{n} a_{i} * x_{i} * x_{i}^{\prime}-\sum_{i=1}^{n} a_{i}<x><x^{\prime}>\right)
$$




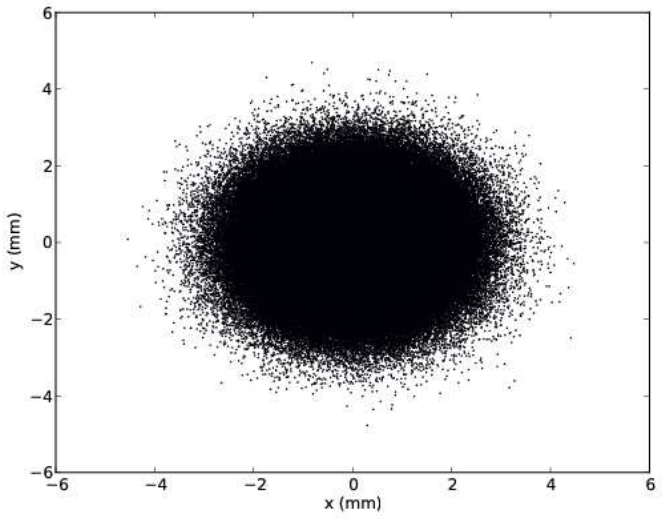

FIG. 2. Two dimensional intensity distribution of inital beam

Then the emittance is calculated by

$$
\epsilon=\sqrt{\left.<x^{2}><x^{\prime 2}>-<x x^{\prime}\right\rangle^{2}}
$$

\section{SIMULATION}

It turns out to be difficult to implement cylindrically non-symmetrical masks in particle tracking code. Therefore, a standalone Python code has been developed to simulate a beam distribution in phase space and to track the distribution through a drift space. Space charge effects are not considered. The statistical error is minimized by generating and tracking 500,000 particles. The density distribution is set to be Gaussian in 4D phase space with standard deviation of $1 \mathrm{~mm}$ for position and 1 mrad for divergence for both planes (numbers are chosen according to the expected BNL ERL beam parameters). In the following, we will try to recover horizontal emittance with the algorithm presented in previous section.

The 2D intensity distribution of the initial beam is shown in Fig. 2. The particle distribution in horizontal phase space is shown in Fig. 3. The projection onto the horizontal plane of the intensity distribution is shown in Fig. 4. The exact horizontal emittance of initial beam is $1.001 \mathrm{~mm} \cdot \mathrm{mrad}$. The vertical multi-slit mask is designed with a slit width of $0.1 \mathrm{~mm}$ and a spacing between slits of $0.9 \mathrm{~mm}$. One of the slits is set to be centered on the beam. The intensity distribution of the sampled beamlets at the mask is shown in Fig. 5. The horizontal emittance of the sampled beam is $0.998 \mathrm{~mm} \cdot \mathrm{mrad}$. This shows that the sampled beam represents the real beam well in terms of emittance.

After propagation in a drift space $(L=0.15 \mathrm{~m})$, the beamlets project onto a profiler (YAG screen for the BNL ERL) with a 2D image shown in Fig. 6.

The projection of the $2 \mathrm{D}$ image at the profiler on the horizontal plane produces a $1 \mathrm{D}$ distribution of multiple quasi-Gaussian peaks. A Python program fits the distribution to multiple Gaussian peaks (a good approxima-

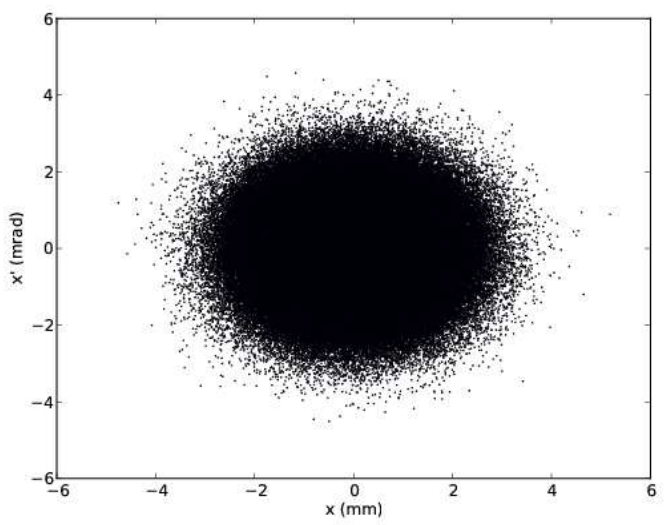

FIG. 3. The particle distribution in horizontal phase space of inital beam

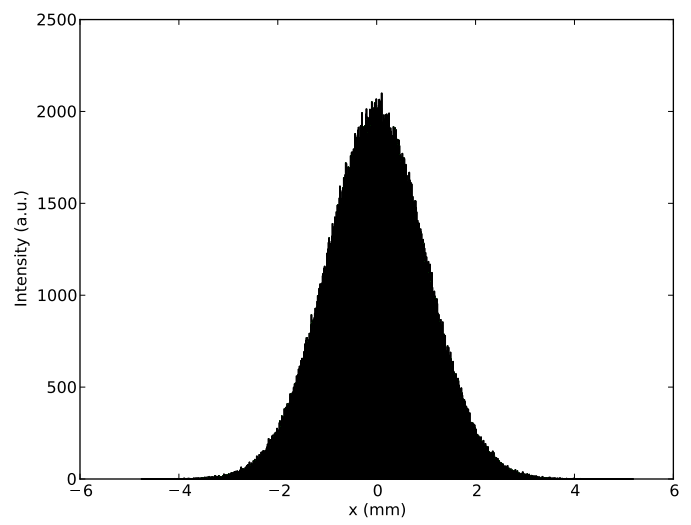

FIG. 4. The horizontal plane projection of intensity distribution

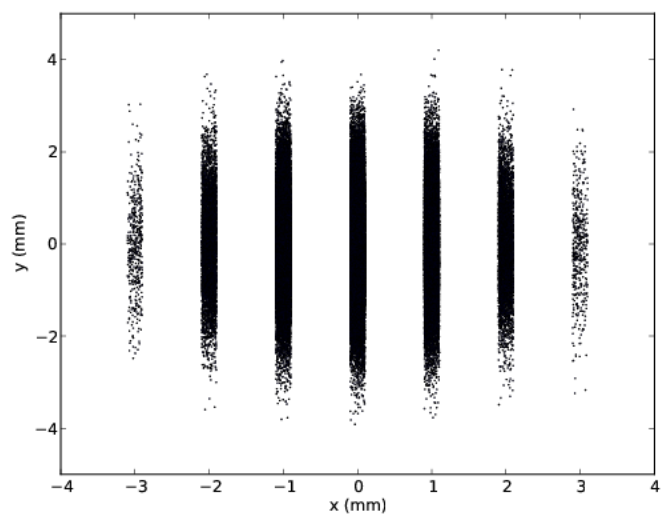

FIG. 5. The intensity distribution of sampled beamlets at the mask 


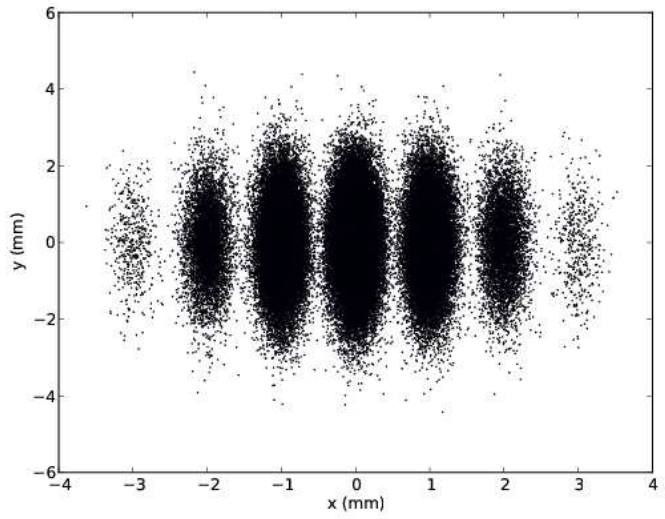

FIG. 6. The intensity distribution of sampled beamlets at the profiler for a drift length of $0.15 \mathrm{~m}$

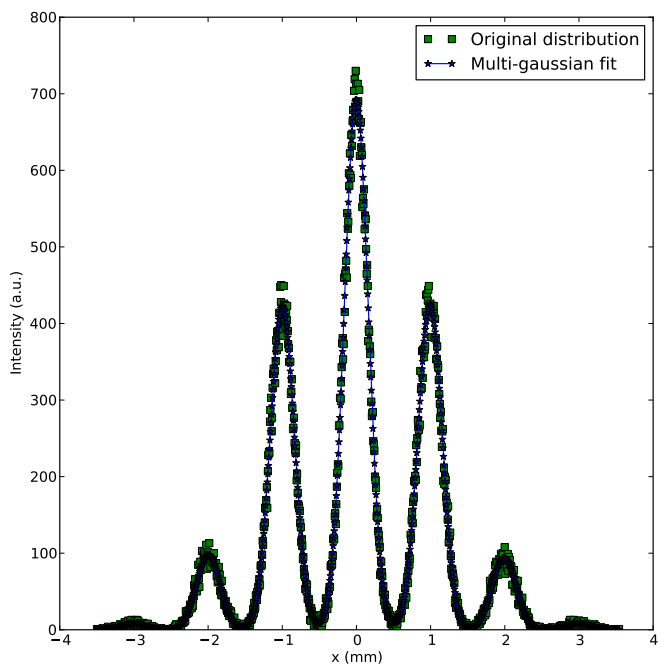

FIG. 7. The horizontal projection of $2 \mathrm{D}$ image on the profiler and its multi-Gaussian fitting for a drift length of $0.15 \mathrm{~m}$

tion for our case with narrow slits). In the process, one finds the intensity of each beamlet, rms width and peak location. The Gaussian fits of the original distributions are shown in Fig. 7. The calculated emittance is 1.067 $\mathrm{mm} \cdot \mathrm{mrad}$.

\section{A. Slit width dependency}

The Gaussian peak assumption and calculation of rms divergence of beamlets are possible due to the fact that the slit width is relatively small. Therefore, the finite slit width may introduce a systematic error in the final determined beam emittance; in principle, the narrower the slit, the closer the measured and real emittances will

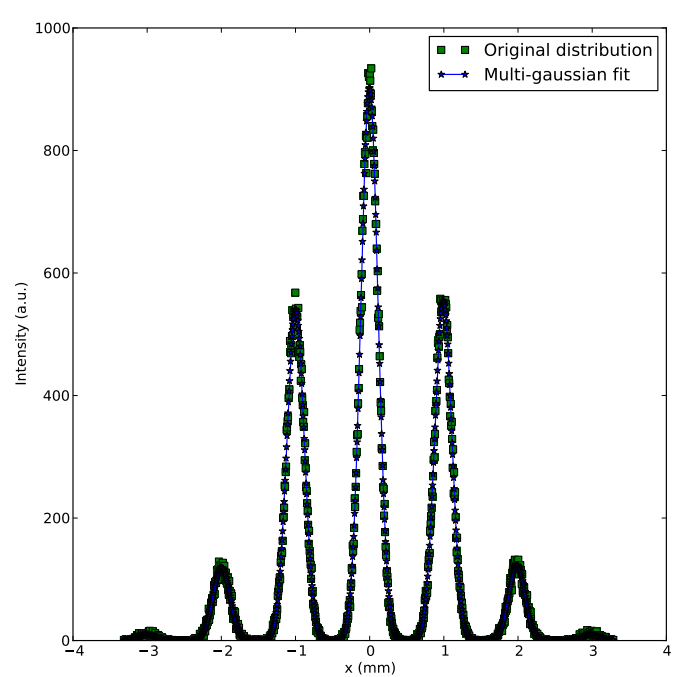

FIG. 8. The horizontal projection and its multi-Gaussian fitting for drift length $100 \mathrm{~mm}$ case

be. We studied the slit width dependency of the retrieved beam emittance with results shown in Table I. In these simulations, the sum of slit width and slit spacing is kept constant at $1 \mathrm{~mm}$ so that total number of beamlets is always seven. Real emittance refers to the emittance of the initial beam, the sampled emittance is the emittance of the beam sampled by multi-slit mask, the simulated emittance is the emittance determined by the algorithm introduced. Deviation refers to the relative difference between simulated emittance and sampled emittance.

The slit width correction has been developed and will be presented in a separate note.

\section{B. Drift length dependency}

Slit widths smaller than $80 \mu m$ are difficult to produce due to mechanical fabrication limitations. For the BNL ERL, the slit width is designed to be $100 \mu \mathrm{m}$. One other parameter which has impact on the simulated emittance is the drift length. The well accepted criteria is that the drift length should be long enough until the beamlets overlap. The following study explores the drift length dependency with results shown in Table II.

The horizontal distribution and its multi-Gaussian fitting for $100 \mathrm{~mm}$ and $230 \mathrm{~mm}$ drift length are shown in Fig. 8 and 9 . The beamlets overlap significantly with each other at a $230 \mathrm{~mm}$ drift length. We were able to determine a more accurate emittance from the fitting at $230 \mathrm{~mm}$ than at $100 \mathrm{~mm}$. We believe this is due to the fact that the slit width contribution is less when the drift length is longer. Therefore, beamlet overlap is acceptable and desirable as long as reasonable fitting can be produced. 
TABLE I. Slit width dependency of simulated emittance

\begin{tabular}{|c|c|c|c|c|c|c|c|}
\hline Slit Width & Slit Spacing & Drift Length & Real Emittance & Sampled Emittance & Simulated Emittance & Deviation \\
\hline $0.05 \mathrm{~mm}$ & $0.95 \mathrm{~mm}$ & $150 \mathrm{~mm}$ & $1.000 \mathrm{~mm} \cdot \mathrm{mrad}$ & $0.993 \mathrm{~mm} \cdot \mathrm{mrad}$ & $0.996 \mathrm{~mm} \cdot \mathrm{mrad}$ & $0.3 \%$ \\
\hline $0.08 \mathrm{~mm}$ & $0.92 \mathrm{~mm}$ & $150 \mathrm{~mm}$ & $1.000 \mathrm{~mm} \cdot \mathrm{mrad}$ & $0.995 \mathrm{~mm} \cdot \mathrm{mrad}$ & $1.009 \mathrm{~mm} \cdot \mathrm{mrad}$ & $1.4 \%$ \\
\hline $0.10 \mathrm{~mm}$ & $0.90 \mathrm{~mm}$ & $150 \mathrm{~mm}$ & $0.998 \mathrm{~mm} \cdot \mathrm{mrad}$ & $0.991 \mathrm{~mm} \cdot \mathrm{mrad}$ & $1.012 \mathrm{~mm} \cdot \mathrm{mrad}$ & $2.1 \%$ \\
\hline $0.12 \mathrm{~mm}$ & $0.88 \mathrm{~mm}$ & $150 \mathrm{~mm}$ & $1.003 \mathrm{~mm} \cdot \mathrm{mrad}$ & $1.006 \mathrm{~mm} \cdot \mathrm{mrad}$ & $1.037 \mathrm{~mm} \cdot \mathrm{mrad}$ & $3.1 \%$ \\
\hline $0.15 \mathrm{~mm}$ & $0.85 \mathrm{~mm}$ & $150 \mathrm{~mm}$ & $1.001 \mathrm{~mm} \cdot \mathrm{mrad}$ & $0.993 \mathrm{~mm} \cdot \mathrm{mrad}$ & $1.037 \mathrm{~mm} \cdot \mathrm{mrad}$ & $4.4 \%$ \\
\hline
\end{tabular}

TABLE II. Drift length dependency of simulated emittance

\begin{tabular}{|c|c|c|c|c|c|c|}
\hline Slit Width & Slit Spacing & Drift Length & Real Emittance & Sampled Emittance & Simulated Emittance & Deviation \\
\hline $0.10 \mathrm{~mm}$ & $0.90 \mathrm{~mm}$ & $100 \mathrm{~mm}$ & $1.002 \mathrm{~mm} \cdot \mathrm{mrad}$ & $1.005 \mathrm{~mm} \cdot \mathrm{mrad}$ & $1.043 \mathrm{~mm} \cdot \mathrm{mrad}$ & $3.8 \%$ \\
\hline $0.10 \mathrm{~mm}$ & $0.90 \mathrm{~mm}$ & $120 \mathrm{~mm}$ & $1.003 \mathrm{~mm} \cdot \mathrm{mrad}$ & $0.997 \mathrm{~mm} \cdot \mathrm{mrad}$ & $1.024 \mathrm{~mm} \cdot \mathrm{mrad}$ & $2.7 \%$ \\
\hline $0.10 \mathrm{~mm}$ & $0.90 \mathrm{~mm}$ & $150 \mathrm{~mm}$ & $1.000 \mathrm{~mm} \cdot \mathrm{mrad}$ & $0.997 \mathrm{~mm} \cdot \mathrm{mrad}$ & $1.013 \mathrm{~mm} \cdot \mathrm{mrad}$ & $1.6 \%$ \\
\hline $0.10 \mathrm{~mm}$ & $0.90 \mathrm{~mm}$ & $180 \mathrm{~mm}$ & $0.998 \mathrm{~mm} \cdot \mathrm{mrad}$ & $0.990 \mathrm{~mm} \cdot \mathrm{mrad}$ & $0.998 \mathrm{~mm} \cdot \mathrm{mrad}$ & $0.8 \%$ \\
\hline
\end{tabular}

\section{SUMMARY}

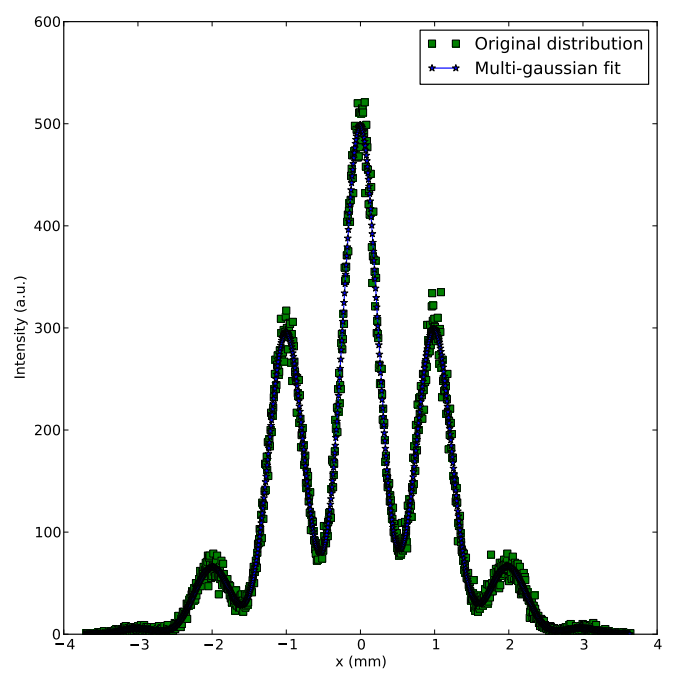

We developed a program for analyzing a multi-slit image for emittance measurement. The functionality was verified in simulations. From these we conclude that the narrower the slit width the better precision of the emittance measurement and that beamlets overlap on the profile screen is to some extent desirable. In the future, we will include space charge effects in the simulation.

FIG. 9. The horizontal projection and its multi-Gaussian fitting for drift length $230 \mathrm{~mm}$ case

[1] P. Piot, J. Song, R. Li, G. Krafft, D. Kehne, K. Jordan, E. Feldl, and J. Denard, in Particle Accelerator Conference, 1997. Proceedings of the 1997, Vol. 2 (IEEE, 1997) pp. 2204-2206.

[2] Y. Li-Xin, D. Ying-Chao, X. Dao, L. Ren-Kai, Q. HouJun, H. Wen-Hui, T. Chuan-Xiang, L. Yu-Zheng, and
C. Jian-Ping, Chinese Physics Letters 25, 1640 (2008). [3] M. Zhang, Fermilab-TM-1988, October(1996). 